\title{
Do Circulating RSV-Genotypes Affect Established Biennial Epidemic Periodicity in Zagreb Region?
}

\author{
Gordana Mlinaric-Galinovic ${ }^{1}$, Dubravko Forcic ${ }^{2}$, Jelena Ivancic-Jelecki ${ }^{2}$, Gordana Vojnovic ${ }^{3}$, \\ Jadranka Bozikov ${ }^{4}$, Robert C. Welliver ${ }^{5}$ \\ ${ }^{1}$ Department of Virology, Croatian National Institute of Public Health and University Medical School of Zagreb, Zagreb, Croatia \\ ${ }^{2}$ Institute of Immunology Zagreb, Zagreb, Croatia \\ ${ }^{3}$ Department of Virology, Croatian National Institute of Public Health, Zagreb, Croatia \\ ${ }^{4}$ Department of Medical Statistics, Epidemiology and Medical Informatics, A. Stampar School of Public Health, \\ Medical School University of Zagreb, Zagreb, Croatia \\ ${ }^{5}$ Division of Infectious Diseases, Department of Pediatrics, Children's Hospital, Oklahoma University Health Sciences Center, \\ Oklahoma City, USA \\ Email: gordana.galinovic@hzjz.hr
}

Received August 13, 2012; revised September 15, 2012; accepted September 24, 2012

\begin{abstract}
Objective: The biennial epidemic pattern of respiratory syncytial virus (RSV) circulation in Croatia has been preserved and could not be related to climatic factors and the predominant RSV subtypes. The possibility that the circulation of different RSV genotypes affect the outbreak cycle in children in Croatia (Zagreb region) over a period of 3 consecutive years was explored in the paper. Methods: The study group consisted of inpatients, aged 0 - 10 years, who were hospitalized with acute respiratory tract infections caused by RSV, in Zagreb, over the period from 1 January 2006 to 31 December 2008. The virus was identified in the nasopharyngeal secretion using direct immunofluorescence method. The virus subtype and genotype was determined by real-time PCR and sequence analysis, respectively. Results: RSV infections identified in 731 children. RSV subtype A caused 399 infections, and subtype B 332. Two subtype A genotypes (NA1 and GA5) and three subtype B genotypes (BA7, BA9 and BA10) were found. During persistent RSV biennial cycles namely four succeeding outbreaks, the new genotype from the previous smaller outbreak persevered into the upcoming larger outbreak. Conclusion: Our molecular-epidemiology study of RSV subtypes and genotypes during calendar months demonstrates that the biennial RSV cycle cannot be fully explained by the dynamic of the predominant circulating genotype of RSV. Other unknown factors account for the biennial cycle of RSV epidemics in Croatia.
\end{abstract}

Keywords: Biennial Cycle; Respiratory Syncytial Virus Types A and B; Genotypes

\section{Introduction}

The biennial pattern of respiratory syncytial virus (RSV) circulation in Croatia, which is similar to that reported in other Central European countries [1-6] has documentedly been preserved for the last 15 years $[1,2,7,8]$. Many earlier studies have attempted to explain the epidemic pattern of RSV activity. We found the anticipated biennial circulation of RSV in the period from January 1, 2006 to December 31, 2008 [9]. Since the two-year periodicity of RSV infections in Croatia could not be related to climatic factors [1], we examined whether this epidemiological characteristic of RSV infections in Croatia could be related to a regular exchange of the two viral subtypes [9]. However, biennial virus cycles were found to be persistent, although the predominant RSV subtype in the first two epidemic waves was subtype $\mathrm{B}$, while in the second two subtype A. Consequently, according to that findings, it may be concluded that neither of the predominant RSV subtypes has an effect on the periodicity of RSV infections in Croatia.

Recent studies have investigated the genotypic pattern of RSV strains during epidemics. The results show a possible correlation between newly emerging genotypes and higher chances for reinfection, possibly eventually leading to a large outbreak $[10,11]$.

In order to determine if the RSV genotypes affect the virus outbreak cycle in Croatia, the paper analyses the molecular epidemic pattern of RSV in children in Croatia (Zagreb region) over a period of 3 consecutive years as a follow-up on and additional molecular examination of RSV isolates from a previous and partially published study $[9,12]$.

\section{Patients and Methods}

The study group consisted of 731 RSV positive inpatients, 
aged 0 - 10 years, who were hospitalized with acute respiratory tract infections, in Zagreb, over the period from 1 January 2006 to 31 December 2008. The majority of patients (696) was taken over from the previous study [9], namely all patients aged $0-5$. An additional 35 individuals hospitalized due to RSV infection in the time period and aged $5-10$ were collected and aggregated. The study was conducted as part of the scientific projects $\# 0005002$ and 005-0053443-3447 (G. M-G), approved by the Ethics Committees of the Croatian National Institute of Public Health (CNIPH).

The virus was identified in the nasopharyngeal secretion (NPS) using a direct fluorescence assay (DFA-Light Diagnostics, Chemicon International, Inc., Temecula, CA) at the Department of Virology, CNIPH [9]. All 731 RSVpositive NPSs were subjected to molecular typing by one-step Real-Time (RT) PCR [9]. RNA was extracted from NPS using a spin column kit (QIAamp DNA Mini Kit; QIAGEN GmbH, Hilden). The N gene of RSV subtype A and B was the target for PCR (using a single-tube RT-PCR kit: TaqMan One-Step RT-PCR Master Mix Reagents Kit; Applied Biosystems, New Jersey, USA). Amplification and detection were performed with a 7500 Real Time PCR System machine (Applied Biosystems) [9]. Nucleotide sequences of RSV subtype A and B strains were obtained from the GenBank and used to construct alignments and phylogenetic trees (the nucleotide sequence spanned bases 5274 to $5543(270 \mathrm{nt})$ of prototype strain $\mathrm{A} 2$; for subtype $\mathrm{B}$ viruses, the sequence corresponded to bases 652 to 981 (330 nt) of BA4128/ 99B, a genotype B prototype strain from Argentina). Nucleotide sequencing was done for 72 strains' $G$ protein gene (the second hypervariable regions HVR2) and the phylogenetic analysis was done for the genotypes found in both viral subtypes [12]. The nucleotide sequences of RSV strains obtained in the study were deposited in GenBank under accession numbers JQ844855JQ844875 [12]. Microsoft Excel was used for data description and graphical presentation.

\section{Results}

Of 731 RSV infections identified in children, subtype A virus caused 399 infections, and subtype B 332. Four patients had a dual RSV infection (subtypes A and B). The further procedure of molecular typing-genome sequencing was applied to examine 41 isolates of subtype A and 31 isolates of subtype B. It was established that subtype A isolates were found in 13 groups of the phylogenetic tree, that consisted of two genotypes (NA1 and GA5). Subtype B isolates were found in 8 groups of the phylogenetic tree or three genotypes (BA7, BA9 and BA10).

The first outbreak was in the spring of 2006. RSV sub- type B generally predominated (105 cases) over subtype A (22 cases). Subtype B genotypes: BA7 (HR3310-06 and HR3604-06) and BA9 (HR2085-06) were found (Figure 1).

The second outbreak was in the winter of 2006/07. RSV subtype B continued to prevail (169 cases) over subtype A (45 cases). Subtype B genotype BA9 (HR14602-06-23 isolates and HR6282-07) and subtype A genotype GA5 (HR263-07, HR2808-07, HR2671-07, HR6010-07, HR4135-07-3 isolates) were found (Figure 1).

The third outbreak was in the spring of 2007. Here RSV subtype A was prominently predominant (174 cases) over subtype B ( 2 cases). Subtype A genotype NA1 (HR16295-07-17 isolates, HR6857-08, HR6931-08-2 isolates) were found (Figure 1).

The fourth outbreak was in the winter of 2007. RSV subtype A again prevailed (158 cases) over subtype B (56 cases). Subtype A genotype NA1 (HR17818-07-2 isolates, HR18543-08-8 isolates, HR19079-08-2 isolates, HR19501-08 and HR20169-08) and subtype B genotype BA10 (HR18724-08-2 isolates, HR18939-08 and HR20806-08) were found (Figure 1).

In summary, the period of study was characterized by 4 epidemic waves of RSV infections: the first, smaller, in the spring of 2006 (genotypes BA7 and BA9), the second, larger, in December 2006/January 2007 (genotypes GA5 and BA9), the third in spring 2008 (genotype NA1), followed by a fourth outbreak beginning in November of 2008 (genotypes NA1 and BA10). During the course of each outbreak novel genotypes of subtypes A and/or B appeared. The genotype from the previous spring/smaller outbreak persisted into the upcoming winter/larger outbreak after an 8-month break (genotype BA9 from the first outbreak continued to the second one and genotype NA1 from the third to fourth outbreak). In the third spring outbreak the newly predominant genotype NA1 appeared. However, the biennial virus cycles were consistently persistent.

\section{Discussion}

The results of our study showed that newly circulating RSV genotypes from the smaller spring outbreak can affect the biennial epidemic periodicity in Zagreb region by triggering the larger winter outbreak in the period of only 8 months (a new genotype belatedly enters the community in spring, and becomes the epidemic strain during the next winter causing a large outbreak, which essentially immunizes the population so that it almost prevents the epidemic for the next year). After that the longer period of 14 months would be needed for the next newly predominant genotype to start the spring outbreak. These findings go hand in hand with the findings of $\mathrm{K}$. T. 
B

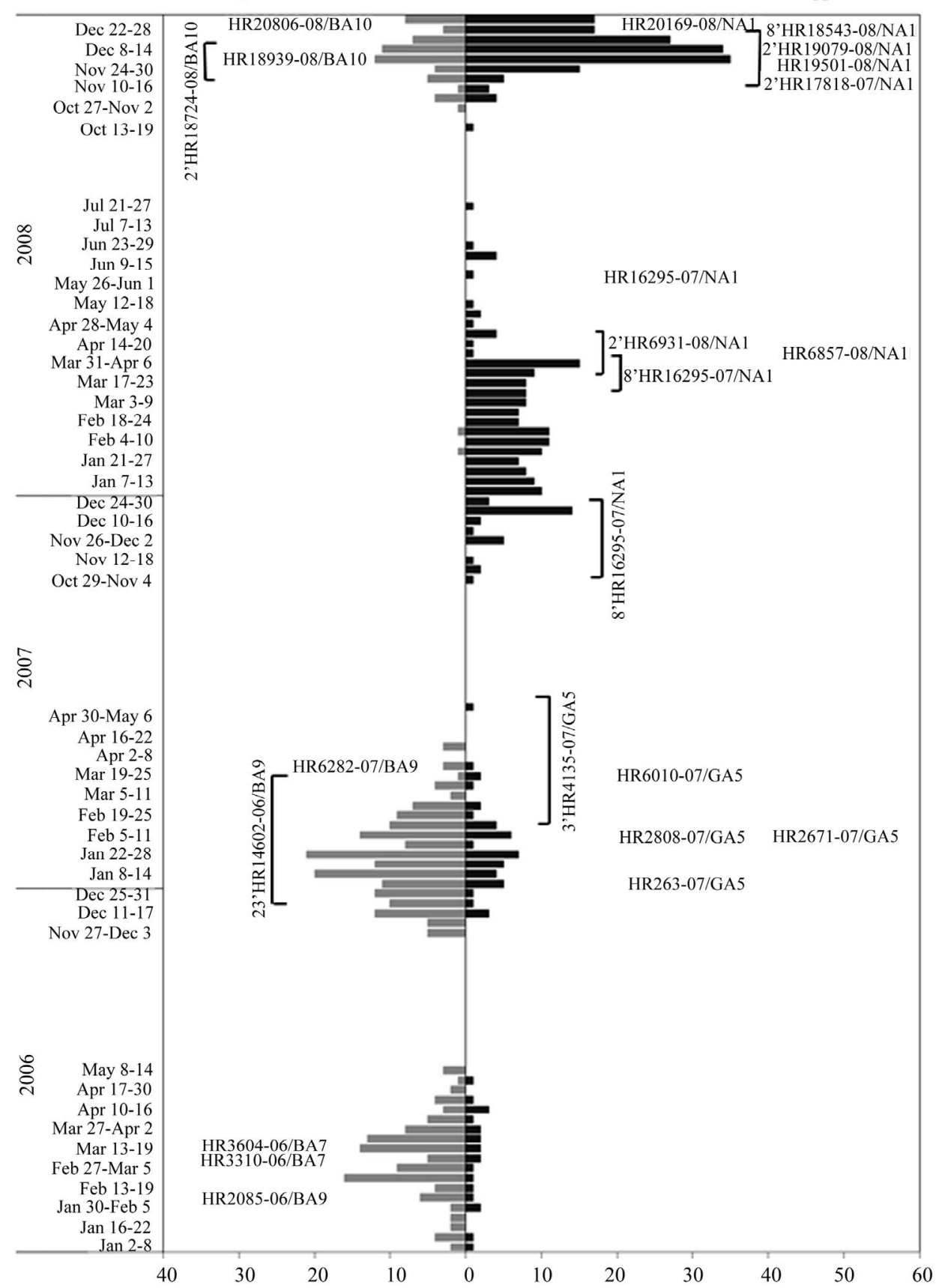

Figure 1. Occurrence of respiratory syncytial virus genotypes (subtype A-right, subtype B-left) by calendar week in the epidemic waves during 2006-2008 for children aged 0 - 10 years (No. 727 + 4 dual infections) in Zagreb, Croatia.

Zlateva, et al. [10] and Y. Shobugawa, et al. [11]. A follow-up study should ensue over the course of several successive years to ascertain with safety the genotypic mechanism of RSV outbreaks. The authors believe that the novel genotypic domination is an important factor for the cyclic occurrence of RSV outbreaks in a moderate climate belt. However, our study showed that viral genotypes did not affect the RSV biennial cycle, as it persisted regardless of the dominant viral genotype. Fur- thermore, the cause of biennial viral cycles in, say, Central Europe, remains to be fully elucidated as opposed to a monophase viral cycle in Belgium, Japan and Great Britain, for example $[10,11,13]$.

In conclusion, authors believe that other factors (besides climatic, biological and behaviorally viral or human), still unknown, may be responsible for these differences in cyclic (monophase and biennial) occurrence of RSV outbreaks. 


\section{Acknowledgements}

This research was carried out as part of the Croatian Ministry of Science, Education and Sports project \#0005002 and \#005-0053443-3447 (G. MG.). The authors thank Renata Sim, DVM, for technical assistance.

\section{REFERENCES}

[1] G. Mlinaric-Galinovic, et al., "The Biennial Cycle of Respiratory Syncytial Virus Outbreaks in Croatia," Virology Journal, Vol. 5, No. 1, 2008, pp. 18-22. doi:10.1186/1743-422X-5-18

[2] G. Mlinaric-Galinovic, et al., "Does the Viral Subtype Influence the Biennial Cycle of Respiratory Syncytial Virus?" Virology Journal, Vol. 6, No. 1, 2009, pp. 133139. doi:10.1186/1743-422X-6-133

[3] E. Terletskaia-Ladwig, et al., "Defining the Timing of Respiratory Syncytial Virus (RSV) Outbreaks: An Epidemiological Study," BMC Infectious Diseases, Vol. 5, No. 1, 2005, pp. 20-27. doi:10.1186/1471-2334-5-20

[4] R. Berner, et al., "Community and Nosocomially Acquired Respiratory Syncytial Virus Infection in a German Paediatric Hospital from 1988 to 1999," European Journal of Pediatrics, Vol. 160, No. 9, 2001, pp. 541-547. doi:10.1007/s004310100801

[5] A. Duppenthaler, et al., "Two-Year Periodicity of Respiratory Syncytial Virus Epidemicsin Switzerland," Infection, Vol. 31, No. 2, 2003, pp. 75-80. doi:10.1007/s15010-002-3124-8

[6] S. W. Aberle, et al., "Biennial Spring Activity of Human Metapneumovirus in Austria," Pediatric Infectious Dis- ease Journal, Vol. 27, No. 12, 2008, pp. 1065-1068. doi:10.1097/INF.0b013e31817ef4fd

[7] G. Mlinaric-Galinovic, et al., "Eleven Consecutive Years of Respiratory Syncytial Virus Outbreaks in Croatia," $\mathrm{Pe}$ diatrics International, Vol. 51, No. 2, 2009, pp. 237-240. doi:10.1111/j.1442-200X.2008.02723.x

[8] G. Mlinaric-Galinovic, et al., "Znacajke Javljanja Epidemije Respiratornim Sincicijskim Virusom U Sezoni 2006/7 U Zagrebačkoj Županiji," Paediatria Croatica, Vol. 53, No. 2, 2009, pp. 49-52.

[9] G. Mlinaric-Galinovic et al., "Analysis of Biennial Outbreak Pattern of Respiratory Syncytial Virus According to Subtype (A and B) in the Zagreb Region," Pediatrics International, Vol. 54, No. 3, 2012, pp. 331-335. doi:10.1111/j.1442-200X.2011.03557.x

[10] K. T. Zlateva, et al., "Subgroup Prevalence and Genotype Circulation Patterns of Human Respiratory Syncytial Virus in Belgium during Ten Successive Epidemic Seasons," Journal of Clinical Microbiology, Vol. 45, No. 9, 2007, pp. 3022-3030. doi:10.1128/JCM.00339-07

[11] Y. Shobugawa, et al., "Emerging Genotypes of Human Respiratory Syncytial Virus Subgroup A among Patients in Japan," Journal of Clinical Microbiology, Vol. 47, No. 8, 2009, pp. 2475-2482. doi:10.1128/JCM.00115-09

[12] D. Forcic, et al., "A Study of the Genetic Variability of Human Respiratory Syncytial Virus in Croatia, 20062008," Journal of Medical Virology, Vol. 84, No. 12, 2012, pp. 1985-1992.

[13] N. L. Goddard, et al., "Timing of Monoclonal Antibody for Seasonal RSV Prophylaxis in the United Kingdom," Epidemiology and Infection, Vol. 135, No. 1, 2007, pp. 159-162. doi: $10.1017 / \mathrm{S} 0950268806006601$ 